\title{
Setzt der Morbi-RSA Anreize für Versorgungsmanagement?
}

EMIN ASGAROV, DIRK HANSEN

Emin Asgarov ist Risikoanalyst bei der kaufmännischen Krankenkasse KKH in Hannover.

Dirk Hansen leitet das Sachgebiet Risikoanalysen bei der $\mathrm{KKH}$ in Hannover

\author{
Seit 2009 erhalten die gesetzlichen Krankenkassen \\ ihre Einnahmen aus dem Gesundheitsfonds über den \\ morbiditätsorientierten Risikostrukturausgleich (RSA). \\ Gesundheitspolitisches Ziel war, dass die Krankenkassen \\ ihre wirtschaftliche Situation durch verbesserte Versorgung \\ steuern können. Dafür wurde der RSA so konstruiert, \\ dass er die prospektiven Folgekosten einer Erkrankung \\ ausgleicht. Der Beitrag zeigt anhand von Realdaten aus \\ zwei Jahren die Ist-Situation bei Deckungsbeiträgen von \\ Hochkostenversicherten und modelliert an einer 10-Jahres- \\ Zeitreihe die Entwicklung von Deckungsbeiträgen. \\ Diskutiert werden auch die Möglichkeiten und \\ Grenzen der Beeinflussung der Versorgung durch die \\ Krankenkasse sowie Änderungsoptionen für den RSA.
}

\section{Einleitung}

Seit 2009 erhalten die gesetzlichen Krankenkassen ihre Einnahmen aus dem Gesundheitsfonds über den morbiditätsorientierten Risikostrukturausgleich (Morbi-RSA). Zusätzlich zu der Berücksichtigung von Alter und Geschlecht sind ausgleichsfähige Krankheiten definiert worden, für die es $\mathrm{Zu}$ weisungen von Einnahmen gibt. Im Gegenzug entfiel der Risikopool, der vor 2009 einen Teil der Ausgaben für Hochkostenversicherte ausglich [Bohlen-Schöning 2008].

Vor 2009 existierte in der gesetzlichen Krankenversicherung bereits ein Risikostrukturausgleich (RSA). Dieser hatte das Ziel, finanzielle Ungleichgewichte durch unterschiedliche Strukturen der Versichertenbestände zwischen den Kassen auszugleichen. Das Grundprinzip bestand in der Berechnung nach Alter und Geschlecht standardisierter erwarteter Ausgaben anhand derer ein Gesamtbeitragsbedarf für die jeweilige Einzelkasse errechnet wurde. Der RSA glich zwischen den realen (einkommensabhängigen) Beitragseinnahmen und dem errechneten (Erwartungs-) Beitragsbedarf aus. Zusätzlich wurden Berufs- und Erwerbsunfähigkeit, Krankengeldanspruch und chronische Erkrankung (Teilnahme an Versorgungsprogrammen) als Risikofaktor berücksichtigt, sowie die Anzahl beitragsfrei mitversicherter Familienmitglieder.

In der Folge gab es einen Finanzfluss von Kassen mit einkommensstarken Versicherten zu Kassen mit einkommensschwachen Versicherten. Größter Nachteil dieses Systems war der Umstand, dass die kassenspezifische Morbidität vor allem der multimorbiden älteren Versicherten - manifestiert durch hohe Ausgaben für Leistungen - nicht ausreichend berücksichtigt wurde [BVA 2008].

Zusätzlich gab es einen „Risikopool“, der einen Teil der Ausgaben für Hochkostenversicherte erstattete. Dieser war durch einen Schwellenwert begrenzt und glich ca. $60 \%$ der Ausgaben oberhalb 
des Schwellenwertes aus. 2008 lag dieser Wert bei $21.352 €$. Dies war vor allem für kleine Kassen vorteilhaft, für die eine geringe Zahl von Hochkostenversicherten eine existenzielle Bedrohung sein kann [Drösler et al. 2011].

Als größter Nachteil dieses RSA wurden die Anreize für eine Risikoselektion gesehen. Hierunter wird das Bestreben der Kassen zusammengefasst, attraktiv für gesunde und unattraktiv für kranke Versicherte am Markt zu agieren mit dem Ziel den Beitragssatz $\mathrm{zu}$ senken und $\mathrm{zu}$ wachsen [Drösler et al. 2011].

\section{Die "Treffsicherheit" des RSA war bis 2009 aufgrund der beschränkten Einbeziehung erklärender Variablen noch relativ gering.}

Die „Treffsicherheit“ des RSA bis 2009 war aufgrund der beschränkten Einbeziehung erklärender Variablen noch relativ gering. Beurteilt am Bestimmtheitsmaß $\mathrm{R}^{2}$, welches für die standardisierten und tatsächlichen Ausgaben auf Individualebene errechnet wurde, lag bei 0,06, wobei1,0 ideal wäre. Unter Einbeziehung des Risikopools erreichte der Koeffizient einen Wert von 0,52. [Drösler et al. 2011]

2009 wurde der morbiditätsorientierte RSA (Morbi-RSA) eingeführt. Dies geschah gleichzeitig mit der Einführung eines einheitlichen Beitragssatzes für alle Kassen, der in einen Gesundheitsfonds eingezahlt wird. Die einzelnen Krankenkassen erhalten nun ihre Einnahmen aus dem Fonds nahezu komplett über den Morbi-RSA. Sofern diese Einnahmen nicht ausreichen um die Kosten zu decken, müssen die Kassen einen Zusatzbeitrag erheben.

Die Geldzuweisungen zur Deckung der Leistungsausgaben (die Verwaltungsausgaben und die dafür zugewiesenen Beträge werden in dieser Arbeit nicht betrachtet) erfolgen im MorbiRSA über standardisierte Beträge nach Alter, Geschlecht und Morbidität (es gibt noch eine Reihe von Sonderregelungen z. B. für Auslandsversicherte). Die Morbidität wird über eine Auswahl von Krankheiten abgebildet, die nach Kriterien wie kostenintensiv, schwerwiegend und chronisch definiert sind. 2009 waren es 106 hierarchisierte Morbiditätsgruppen (HMG). Aufgrund von jährlichen Korrekturen ist die Anzahl der HMG mittlerweile auf 155 angewachsen. Das Gütemaß der Zielgenauigkeit gemessen am Bestimmtheitsmaß $\mathrm{R}^{2}$ lag im MorbiRSA 2009 bei 0,2 [Drösler et al. 2011]. Durch die Änderungen der Krankheitsklassifikation der Jahre 2010 bis 2013 liegt das $\mathrm{R}^{2}$ derzeit bei 0,24 (CPM 0,24, MAPE 1.867,77 €) [BVA 2012].

An dieser Stelle setzte in der Vergangenheit die Großteil der Kritik an: die Verteilungswirkung sei zwar deutlich

\section{Abkürzungen}

$\begin{array}{ll}\text { A1, C1-C5 } & \text { Teilgruppen der Hochkostenkohorten, werden in Abschnitt 5.5 erläutert } \\ \text { BB } & \text { Beitragsbedarfswerte } \\ \text { BB-LA } & \text { Differenz zwischen Beitragsbedarf und Leistungsausgaben, Deckungsbeitrag } \\ \text { BBVJ } & \text { Beitragsbedarf je Versichertenjahr (annualisiert) } \\ \text { BVA } & \text { Bundesversicherungsamt } \\ \text { CPM } & \text { Cumming's Prediction Measure (standardisiertes statistisches Gütemaß) } \\ \text { COPD } & \text { Chronic obstructive pulmonary disease } \\ \text { GKV } & \text { Gesetzliche Krankenversicherung } \\ \text { HK } & \text { Hochkostenkohorte } \\ \text { HN } & \text { Hochnutzer (synonym für Hochkostenversicherter) } \\ \text { HMG } & \text { Hierarchisierte Morbiditätsgruppe } \\ \text { ICD } & \text { International Classification of Diseases (internat. Diagnoseschlüssel) } \\ \text { LA } & \text { Leistungsausgaben } \\ \text { LAVJ } & \text { Leistungsausgaben je Versichertenjahr (annualisiert) } \\ \text { MAPE } & \text { Mittlerer absoluter Prognosefehler (mean absolute prediction error) } \\ \text { RSA } & \text { Risikostrukturausgleich } \\ \text { RSAV } & \text { Risikostruktur-Ausgleichsverordnung }\end{array}$

besser als (hauptsächlich über Alter und Geschlecht) im Alt-RSA, aber noch deutlich hinter dem Wert unter Einbeziehung des Risikopools. Die Gegenargumentation ist primär politisch: Es sollen Anreize für die Kassen bestehen, ihre Ausgaben durch Versorgungsangebote und die Unterstützung der Versicherten mittels eines Versorgungsmanagements zu senken. ${ }^{1}$ Außerdem sei eine Reduzierung der Anzahl der Krankenkassen gewünscht [Schmidt 2009], so dass ein Schutz sehr kleiner Kassen über Instrumente wie den Risikopool keine Priorität genießt. Zusätzlich wurde der Organisationsaufwand für den Risikopool im Verhältnis zur Wirkung aufgeführt.

\section{Forschungsfrage}

Der Gesetzgeber hat also mit der Ausgestaltung des Morbi-RSA den Krankenkassen einen Anreiz gesetzt, ihre wirtschaftliche Situation z.B. über ein optimiertes Versorgungsmanagement steuern zu können. Dafür wurde der RSA „prospektiv“ konstruiert [BohlenSchöning, 2008]. ${ }^{2}$ Gelingt es den Kassen im Folgejahr die Versorgung zu verbessern und die Ausgaben zu senken, sollen sie bessere Deckungsbeiträge erzielen können.

Diese Arbeit untersucht anhand der ersten beiden Jahre mit Morbi-RSADaten (2009 und 2010) die Verläufe von Deckungsbeiträgen und geht der Frage nach, wie groß eine Absenkung

1 Der Morbi-RSA gleicht die prospektiven Folgekosten der Erkrankungen aus. Dazu werden für die Versicherten die prospektiven Folgekosten des Vorjahres im Ausgleichsjahr ermittelt. Das gesamte Zuwendungsvolumen legt ein Schätzerkreis fest. Abweichungen zwischen tatsächlichen Ausgaben und Zuwendungsvolumen werden mitgliederbezogen umgelegt.

2 §31 RSAV: „Die Auswahl des Versichertenklassifikationsmodells ... und seine Anpassung an die Gegebenheiten der gesetzlichen Krankenversicherung haben so zu erfolgen, dass keine Anreize für medizinisch nicht gerechtfertigte Leistungsausweitungen geschaffen und Anreize zur Risikoselektion vermieden werden. Das ... an die gesetzliche Krankenversicherung angepasste Versichertenklassifikationsmodell ist an Hand von 50 bis 80 Krankheiten zu filtern und prospektiv auszugestalten. Bei der Auswahl der ... Krankheiten sollen insbesondere Krankheiten mit schwerwiegendem Verlauf und kostenintensive chronische Krankheiten, bei denen die durchschnittlichen Leistungsausgaben je Versicherten die durchschnittlichen Leistungsausgaben aller Versicherten um mindestens 50 vom Hundert übersteigen, berücksichtigt werden. Die Krankheiten sollen eng abgrenzbar sein." 
Tabelle 1: Größe der Hochkosten-Kohorten (HK)

\begin{tabular}{|l|c|c|c|c|c|c|}
\hline & HK2005 & HK2006 & HK2007 & HK2008 & HK2009 & HK2010 \\
\hline Anzahl & 87.709 & 87.698 & 96.267 & 95.694 & 104.709 & 105.426 \\
\hline
\end{tabular}

Quelle: Eigene Darstellung

der Leistungsausgaben durch bessere Versorgung sein müsste, um positive Deckungen zu erzielen. Am effizientesten ist der Ansatz aus ökonomischer Sicht bei Versicherten mit hohen Leistungsausgaben. Daher konzentriert sich die Untersuchung auf Hochkostenversicherte. Eine weitere Zielsetzung ist, die Auswirkungen des Wegfalls des Risikopools abzuschätzen. Auch für diese Fragestellung bietet sich die Untersuchung von Deckungsbeiträgen für Hochkostenfälle an.

\section{Material und Methoden}

Die untersuchten Daten entstammen dem Versichertenbestand einer Ersatzkasse mit bundesweiter Öffnung aus den Jahren 2005 bis 2010. Die Hochkostenversicherten wurden definiert, in Jahres-Kohorten aufgeteilt und mit dem Leistungsverbrauch in Zusammenhang gebracht. Für die Berechnung einer Langzeitreihe der Deckungsbeiträge mussten die Einnahmen aus dem Morbi-RSA simuliert werden. Dafür wurden die Stabilität der Einnahmen für Kohorten von Hochkostenversicherten einerseits und die Inflation (jährliche Zunahmerate) andererseits anhand vorliegender Daten aus 2009 und 2010 untersucht und die erforderlichen zeitlichen Vorwärts- und Rückwärtsberechnungen mittels eines linearen Auf-/Abzinsungsverfahrens durchgeführt.

\section{Der Deckungsbeitrag unter Morbi-RSA-Bedingungen}

Die Differenz zwischen den Pro-KopfEinnahmen über den Morbi-RSA und den anfallenden Pro-Kopf-Leistungsausgaben wird als Deckungsbeitrag bezeichnet. Dabei werden in dieser Arbeit nur die auf die nach RSA-Definition „standardisierten Leistungsausgaben“

fallenden Komponenten betrachtet. Die Einnahmen wurden mittels GroupingVerfahren nach der im jeweiligen Jahr gültigen RSA-Zuordnung individualisiert und mit der Bezeichnung „Beitragsbedarf" versehen. Der Deckungsbeitrag wird in $€$ angegeben. Die Kennzahl gibt Auskunft darüber, inwiefern die individuellen Ausgaben für Leistungen durch die individualisierten RSA-Einnahmen gedeckt werden. Sind die Leistungsausgaben vollständig durch Einnahmen gedeckt, beträgt der Deckungsbeitrag $0 €$. Sind die Ausgaben höher als die Einnahmen, wird der Deckungsbeitrag negativ bzw. umgekehrt.

\section{Hochkostenversicherte und Phasen des Leistungsverbrauches}

Unter Hochkostenversicherten werden hier die 5\% des Versichertenbestandes mit den höchsten Leistungsausgaben verstanden. Im vorliegenden Datenbestand liegen die Pro-Kopf-Ausgaben dieser Versicherten bei ca. $8.000 €$ p. a. und darüber. Die Hochkostendefinition wurde so gewählt, um in Teilgruppen eine für statistische Aussagen ausreichende Menge von Versicherten zu erhalten. Mit einer Definition entsprechend des Risikopools (21.352 €) wären in den relevanten Teilpopulationen nur wenige Versicherte übrig geblieben. In Einzelfällen können Leistungsausgaben mehrere Mio. $€$ p. a. ausmachen. Selten bewegen sich die Leistungsausgaben eines individuellen Versicherten permanent auf so hohem Niveau. In der Regel verzeichnet man in einem Jahr mit Eskalation einer Erkrankung (z. B. Amputation bei Diabetes, Herzinfarkt) oder mit einem gravierenden Unfallereignis einen „Peak“ im Leistungsverbrauch, um in den Folgejahren wieder abzusinken. Statistisch betrachtet befindet sich die Mehrheit der Versicherten, bei denen man in einem

Tabelle 2: Kategorisierung der bekannten Beitragsbedarfswerte (BB)

\begin{tabular}{|l|c|c|c|c|c|c|}
\hline BB 2010 (€) & 6.947 & 7.152 & 7.153 & 7.488 & 8.375 & 6.985 \\
\hline Kohortenjahr & 2005 & 2006 & 2007 & 2008 & 2009 & 2010 \\
\hline Jahr nach Eskalation & $\mathbf{5}$ & $\mathbf{4}$ & $\mathbf{3}$ & $\mathbf{2}$ & $\mathbf{1}$ & $\mathbf{0}$ \\
\hline
\end{tabular}

Quelle: Eigene Darstellung

\section{Definitionen}

Deckungsbeitrag $=($ Beitragsbedarf pro Kopf $)-$ (Individuelle Leistungsausgaben pro Kopf) Beitragsbedarf $=$ Individualisierte Einnahmekomponenten gemäß RSA für die standardisierten Leistungsausgaben auf Basis der Versichertendaten des Berechnungsjahres und der Krankheitsdaten des Vorjahres

Leistungsausgaben $=$ RSA-relevante NettoLeistungsausgaben des Berechnungsjahres u.a. um Erstattungen vermindert, individuell zugeordnet, teilweise pauschalisiert.

bestimmten Jahr einen Hochkostenstatus feststellt, in einer Phase der Eskalation. In dieser Arbeit wird der Begriff Eskalation nicht medizinisch verwendet, sondern statistisch-ökonomisch. Das Eskalationsjahr ist hier das Jahr, in dem die Versicherten einen bestimmten Schwellenwert an Ausgaben überschritten haben. Vor diesem Jahr und danach stellt man in der Regel niedrigere jährliche Ausgaben fest (Ausnahme sind zum Beispiel Kleinkinder, s.u.). Die Phasen vor, während und nach der Eskalation spielen für die Langfrist-Modellierung (s. Abschnitt 5.5) eine wichtige Rolle.

\section{Kohortenbildung}

Aus dem Versichertenbestand der wurde für die Jahre 2005 bis 2010 das zu diesem Zeitpunkt jeweils oberste 5\% Perzentil mit den höchsten Leistungsausgaben ausgewählt und daraus eine Hochkostenkohorte gebildet. Aufgrund der Sterbefälle werden diese Kohorten in der Regel - sofern keine besonderen Veränderungen des Versichertenbestandes auftraten - mit jedem Jahr zurück immer kleiner. Somit ist die Kohorte, die 2006 hohe Ausgaben hatte, heute $\mathrm{N}=87.698$ groß, die Kohorte aus dem Jahr 2010 ist mit $\mathrm{N}=105.426$ die größte (Tabelle 1).

Die Leistungsausgaben betragen hier etwa $8.000 €$ und mehr pro Kopf und pro Jahr. In den Leistungsausgaben sind die Ausgaben aller ambulanten und stationären Sektoren enthalten. Die Ausgaben für einige Leistungen wurden pauschal zugeordnet (ambulante Arztleistungen, konservierend-chirurgische Leistungen des zahnärztlichen Bereiches). Um unterjährige Versicherungsverhältnisse zu berücksichtigen wurden die Ausgaben pro Versichertentag berechnet und auf 365 Versichertentage für ein Versichertenjahr komplettiert. 


\section{Simulation des Beitragsbedarfes}

Für die Untersuchung einer Langzeitreihe wären die Deckungsbeiträge von 2005 bis 2010 erforderlich gewesen. Es lagen jedoch erst für zwei Jahre (2009 und 2010) Informationen zu den Beitragsbedarfen („Einnahmen“) aus dem Morbi-RSA vor. Also bestand die Notwendigkeit, die Vorjahre nachzustellen - zu simulieren. Es wurde folgender Ansatz zur Simulation von Beitragsbedarfen gewählt: Zunächst wurden die vorliegenden (bekannten) Pro-Kopf-Beitragsbedarfe bezüglich des zeitlichen Abstandes zum Eskalationsjahr kategorisiert. Die Werte für eine Kategorie (z. B. „ein Jahr vor der Eskalation“) wurden als repräsentativ angenommen. Dadurch werden Klassifikationsänderungen von 2009 auf 2010 berücksichtigt, aber nicht mehr für die Zeit danach. Auf diese Weise erhält man repräsentative Beitragsbedarfswerte von einem Jahr vor der Eskalation bis 5 Jahre nach der Eskalation (Tabelle 2). Es wird also der Beitragsbedarf für das Jahr 2010 für die Versicherten berechnet, die im Jahr 20052010 eine „Eskalation“ hatten. Z. B. für die Versicherten, die im Jahr 2005 eine Eskalation hatten, beträgt der Beitragsbedarf im Jahr $20106.947 €$ ).

Dabei ist allerdings der inflationsbedingte Anstieg der Leistungsausgaben nachzubilden. Er betrug im Zeitraum 2005 bis 2009 zwischen 4\% und 6\% und wurde daher hier gleichmäßig mit $5 \%$ angesetzt. Die modellierten Beitragsbedarfe wurden entsprechend erhöht oder abgesenkt (s. Tabelle 3 ).

\section{Unterteilung in Teilgruppen}

Die Hochkostenkohorten sind insofern heterogen, dass sie sich aus sehr unterschiedlichen Krankheitsbildern, Schweregraden der Erkrankung sowie akuten und chronischen Erkrankungen zusammensetzen. Die in den Abschnitten 6.1 bis 6.5 dargestellten Verläufe zeigen stets die Überlagerung sehr unterschiedlicher Versorgungssituationen. Von Interesse ist daher, ob es homogene Untergruppen gibt, deren Deckungsverläufe charakteristisch sind oder die das Gesamtbild besonders stark prägen. Da eine Unterteilung in einzelne Erkrankungen nach dem ICD-Katalog für diese Untersuchung zu aufwändig gewesen wäre (als problematisch erwies sich die Multimorbidität der überwiegenden Anzahl der Hochkostenversicherten), wur-

Tabelle 3: Inflationsbereinigung bei den simulierten Beitragsbedarfen

\begin{tabular}{|l|c|c|c|c|c|c|}
\hline Korrektur zum Jahr & 2005 & 2006 & 2007 & 2008 & 2009 & 2010 \\
\hline $\begin{array}{l}\text { Korrekturfaktor für den } \\
\text { Beitragsbedarf }\end{array}$ & $75 \%$ & $80 \%$ & $85 \%$ & $90 \%$ & $95 \%$ & $100 \%$ \\
\hline
\end{tabular}

Quelle: Eigene Darstellung

de nach geeigneten Klassifizierungen zur Beschreibung homogener Teilgruppen gesucht. Folgende Kriterien wurden dafür benannt:

- Ausreichende Gruppengröße für statistische Stabilität

- Homogenität bezüglich der zeitlichen Dimension des Kostenrisikos (Chronizität)

- Kostenintensiv

- Schwerwiegend (hohe Hospitalisierungsrate)

- Berücksichtigung des Alters

Von den Gesamtkohorten wurden zunächst die Kleinkinder mit einem Lebensalter bis einschließlich 5 Jahren abgespalten und als erste Teilgruppe definiert („Infant“). Bei dieser Gruppe dominieren Frühgeburten und Kleinkinder mit schwerwiegenden angeborenen Defekten.

Als Nächstes wurden Versicherte mit einer Pflegestufe selektiert („Pflege“). Die Pflegestufe bietet sich als Beschreibungsmerkmal an, welches dauerhafte Einschränkungen von körperlichen Funktionen und Mobilität in Kombination mit einem höheren Alter vereinigt. Voruntersuchungen wiesen bereits darauf hin, dass Versicherte mit einer Pflegestufe überdurchschnittlich negative Deckungsbeiträge aufweisen.

Im dritten Schritt wurden die verbliebenen Personen mit schwerwiegenden und/oder kostenintensiven chronischen Erkrankungen abgespalten („Chroniker“).

Damit verbleibt ein verhältnismäßig kleiner Rest, der durch Akutereignisse (Unfälle, Infektionen) geprägt ist. Die

Tabelle 4: Eckdaten zu den Untergruppen (ohne Verstorbene)

\begin{tabular}{|l|c|c|c|}
\hline Gruppe & Menge 2009 & Anteil (\%) & Sterberate (\%) \\
\hline Infant & 2.994 & 3,1 & 1,4 \\
\hline Pflege & 18.294 & 18,9 & 27,4 \\
\hline Chroniker (C2-C5) & 72.232 & 74,6 & 3,7 \\
\hline Akut (A1 und C1) & 3.337 & 3,4 & 0,9 \\
\hline Gesamt & 96.857 & 100,0 & 8,0 \\
\hline
\end{tabular}

Quelle: Eigene Darstellung
Teilgruppen weisen aufgrund dieser Art der Aufteilung keine Überlappungen auf. Tabelle 4 gibt Auskunft über das Mengengerüst und die Sterberaten in den Untergruppen.

Die leichten chronischen Erkrankungen (C1: Erkrankungen die chronisch sind, aber weder kostenintensiv noch schwerwiegend (z. B. Struma)) wurden der Gruppe „Akut“ (A1) zugeordnet (Gruppengröße und -homogenität). Es ist davon auszugehen, dass die Ausgaben dieser Versicherten weniger durch die chronischen Erkrankungen als durch Akutereignisse hervorgerufen werden. Die Gruppe schwerer erkrankten Chroniker wurde aufgrund der Größe in weitere Untergruppen zerlegt:

C2: Erkrankungen die chronisch sind und kostenintensiv aber nicht schwerwiegend (z. B. Diabetes mellitus)

C3: Erkrankungen die chronisch sind und schwerwiegend aber nicht kostenintensiv (z. B. Luxation des Hüftgelenkes)

C4: Erkrankungen mit dem Status kostenintensiv und schwerwiegend in unterschiedlichen Diagnosen (z. B. Diabetes plus Hüftgelenkluxation)

C5: Erkrankungen mit Diagnosen die sowohl kostenintensiv als auch schwerwiegend sind (z. B. Schizophrenie) 
Tabelle 5: Anzahl der Deckungsbeitragswerte für die Modellierung des Langfristprofils.

\begin{tabular}{|l|c|c|c|c|c|c|c|c|c|c|c|}
\hline & \multicolumn{3}{|c}{ Vor } & \multicolumn{3}{c|}{ Eskalation } \\
\hline $\begin{array}{l}\text { Zeitlicher Abstand vom } \\
\text { Eskalationsjahr (Jahre) }\end{array}$ & -5 & -4 & -3 & -2 & -1 & 0 & 1 & 2 & 3 & 4 & 5 \\
\hline Anzahl verfügbarer Werte & 1 & 2 & 3 & 4 & 5 & 6 & 5 & 4 & 3 & 2 & 1 \\
\hline
\end{tabular}

Quelle: Eigene Darstellung

Die Klassifizierung der Chroniker wurde in Anlehnung an die Krankheitsauswahl des BVA vorgenommen [BVA 2010].

\section{Modellierung des Langfristprofils der Deckungsbeiträge}

Mit der in Abschnitt 5.4 vorgenommenen Simulation des Beitragsbedarfes lagen nunmehr viele Informationen über Deckungsbeiträge der Hochkostenkohorten mit unterschiedlich großem zeitlichem Abstand zum Eskalationsjahr vor. Für die Kohorte aus 2005 repräsentiert der simulierte Deckungsbeitrag für 2010 die Zeit „5 Jahre nach der Eskalation“. Für die Kohorte aus 2010 repräsentiert der simulierte Deckungsbeitrag aus 2005 die Zeit „5 Jahre vor der Eskalation“. Die Werte der Kohorten aus 2010 und 2009 für die Jahre 2006 bzw. 2005 repräsentieren die Zeit „vier Jahre vor der Eskalation“. Mittels der simulierten Werte lässt sich also ein Zeitraum von 5 Jahren vor bis fünf Jahre nach der Eskalation beschreiben - eine Zeitspanne über 10 Jahre. Für bezüglich der Eskalation zeitlich äquidistante Werte der Deckungsbeiträge (z. B. 1 Jahr nach Eskalationsjahr) wurden Mittelwerte (pro-Kopf-Mittelwert der jeweiligen Kohorte) gebildet und zu einer 10-Jahres-Zeitreihe zusammengesetzt. Tabelle 5 gibt Auskunft über die Wertemengen. Für das Eskalationsjahr (= Hochkostenjahr, in Tabelle 5 die fett gedruckte mittlere Spalte) liegen von sechs Kohorten (2005 bis 2010) die realen Deckungsbeiträge vor. Für den Zeitpunkt fünf Jahre vor Eskalation (in Tabelle 5 ganz links) gibt es nur eine Kohorte mit realen Deckungsbeiträgen (2005).

Während die Werte um das Eskalationsjahr also mehrfach besetzt sind, wird die Datenmenge zu den Rändern dünner. Daher sind Interpretationen bezüglich der größeren zeitlichen Abstände von der Eskalation mit Vorsicht vorzunehmen.

\section{Ergebnisse}

\section{Zeitreihe der Ausgaben für die Kohorten}

Für diese Untersuchung wurden die jeweils teuersten $5 \%$ des Versichertenbestandes aus den Jahren 2005 bis 2010 selektiert. Die Größe der Kohorten ist für zurückliegende Jahre aufgrund der Sterbefälle kleiner und beträgt zwischen
88.000 und 105.000 Versicherte (Tabelle 1). Darüber hinaus wurden die Ausgaben dieser 6 Kohorten auch für alle übrigen Jahre dieser Periode 2005 bis 2010 ermittelt. Als Zeitreihe aufgetragen erkennt man jeweils deutlich das Identifikationsjahr als Hochkostenfall, welches als „Peak“ aus der Zeitreihe herausragt.

Die Kostenverläufe der einzelnen Kohorten sind sehr ähnlich. Allerdings ist ein Trend zu höheren Ausgaben zu verzeichnen. Dieses Anwachsen ist vorrangig auf den Inflationseffekt (Preissteigerungen bei Leistungen) zurück zu führen. Fusions- und marktbedingte Änderungen des Versichertenbestandes haben ab 2009 einen geringen Einfluss auf den Ausgabenanstieg.

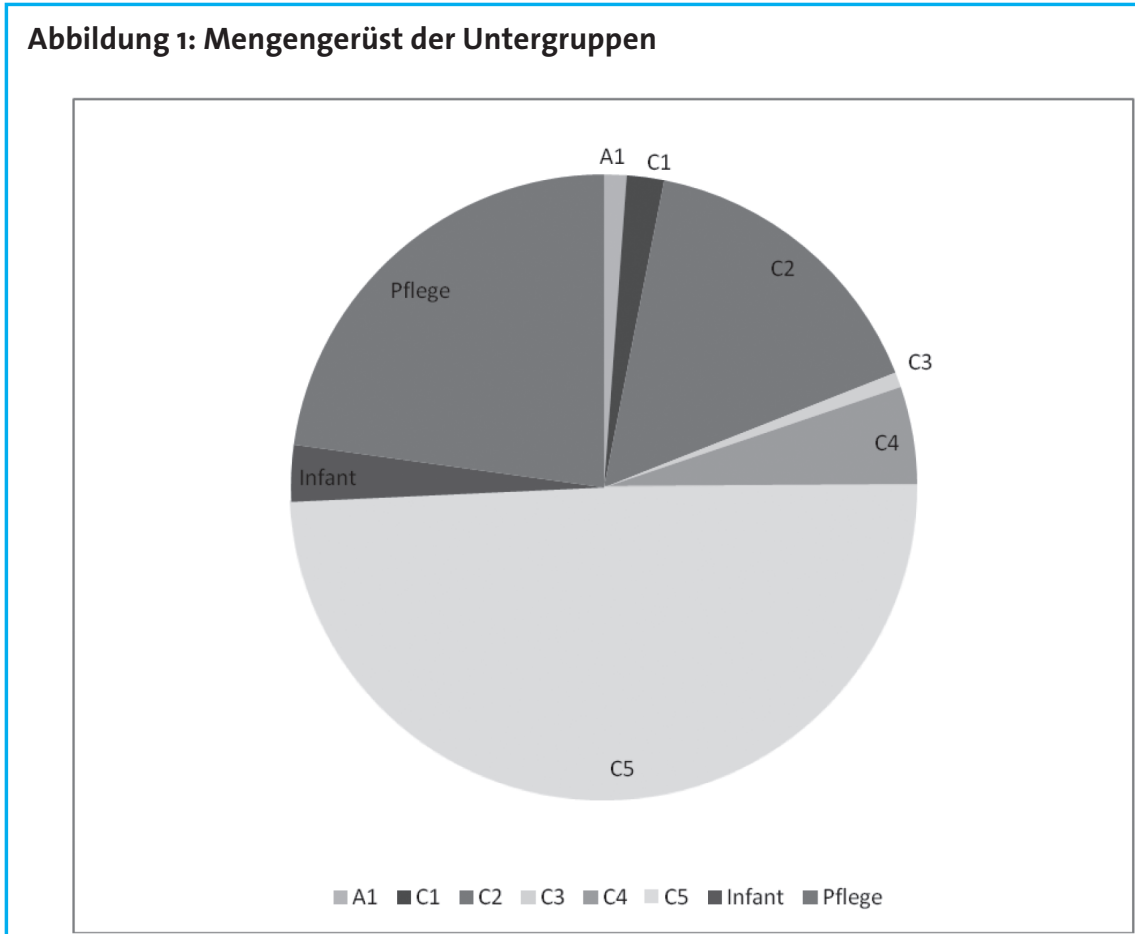

Abbildung 2: Zeitreihe der Leistungsausgaben für die sechs Kohorten

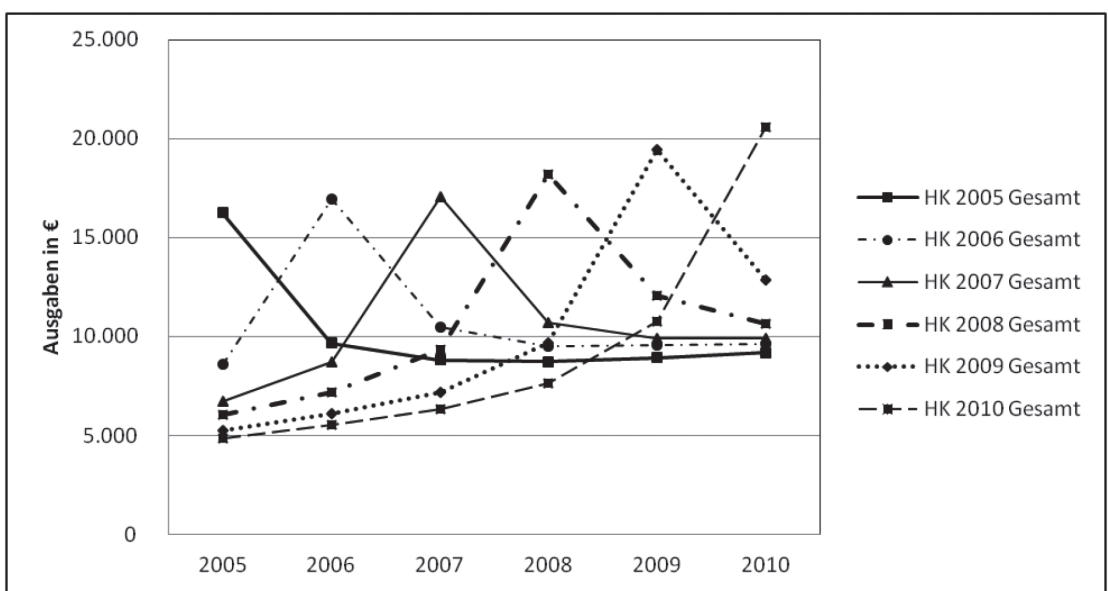




\section{Einfluss der Sterbefälle}

Während die Sterbequote im gesamten Versichertenbestand ca. $1 \%$ beträgt, liegt sie bei den Hochkostenkohorten aufgrund der Morbidität höher. Die unterschiedlichen Kohorten haben im Eskalationsjahr Sterbequoten zwischen 7 und $8 \%$.

Da die Sterbefälle in der Regel (sowohl bei chronischen als auch bei Akutereignissen) mit hohen Leistungsausgaben verbunden sind, wurde die Auswirkung der Sterbefälle auf die Kostenverläufe untersucht. Dabei besteht aufgrund der Vergleichbarkeit die Notwendigkeit, die aufgrund des Versterbens unterjährig anfallenden Kosten auf ein volles Jahr zu normieren. Hier besteht die Gefahr einer systematischen Überschätzung. Die mittleren annualisierten Ausgaben für
Tabelle 7: Beitragsbedarf und Leistungsausgaben aus 2009 für die verschiedenen Hochkostenkohorten (HK)

\begin{tabular}{|l|r|r|r|r|r|r|}
\hline LAVJ 2009 & HK 2005 & HK 2006 & HK 2007 & HK 2008 & HK 2009 & HK 2010 \\
\hline BBVJ 2009 & $6.641 €$ & $-9.603 €$ & $-9.966 €$ & $-12.149 €$ & $-19.493 €$ & $-10.829 €$ \\
\hline LAVJ 2010 & $-9.216 €$ & $-9.687 €$ & $6.956 €$ & $8.146 €$ & $6.614 €$ & $5.946 €$ \\
\hline BBVJ 2010 & $6.947 €$ & $7.152 €$ & $7.153 €$ & $7.488 €$ & $8.375 €$ & $6.985 €$ \\
\hline
\end{tabular}

Quelle: Eigene Darstellung
Sterbefälle liegen bei den Hochkostenversicherten pro Kopf zwischen 30.000 und $40.000 €$ p. a (Abb. 3). Die Annualisierung macht hier - bei den Hochkostenkohorten - eine Erhöhung von ca. $15.000 €$ Ausgaben pro Sterbefall aus.

Aufgrund der geringen Zahl beeinflussen die Sterbefälle die Verläufe der

\section{Abbildung 3: Kostenverläufe der annualisierten Sterbefälle}

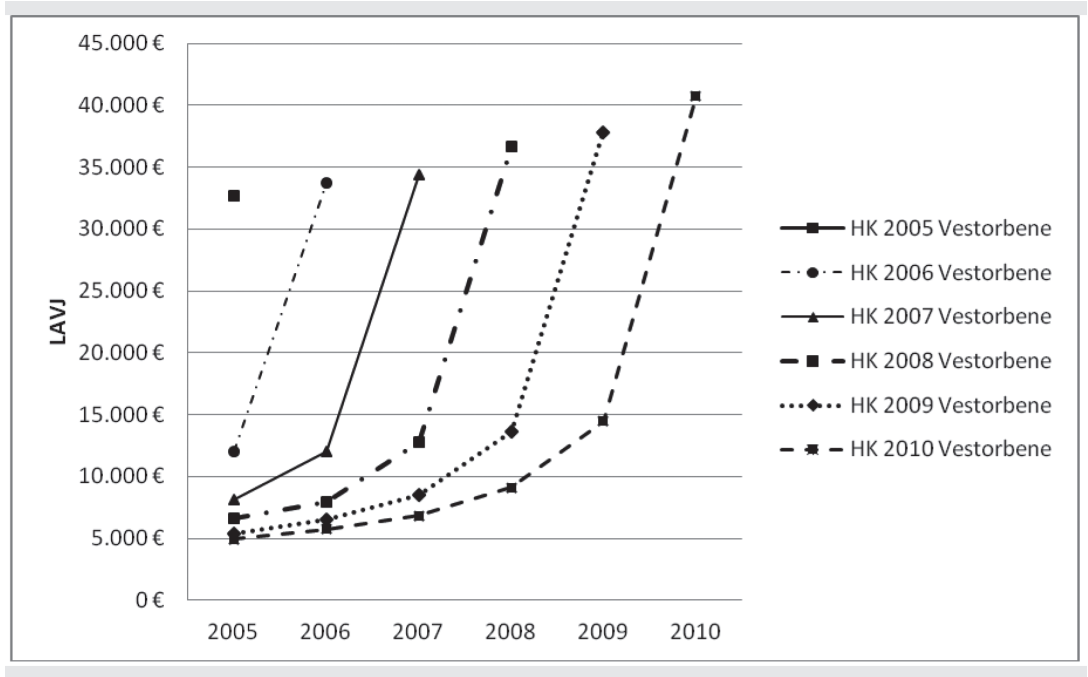

Abbildung 4: Einfluss der Sterbefälle auf die Hochkostenkohorten

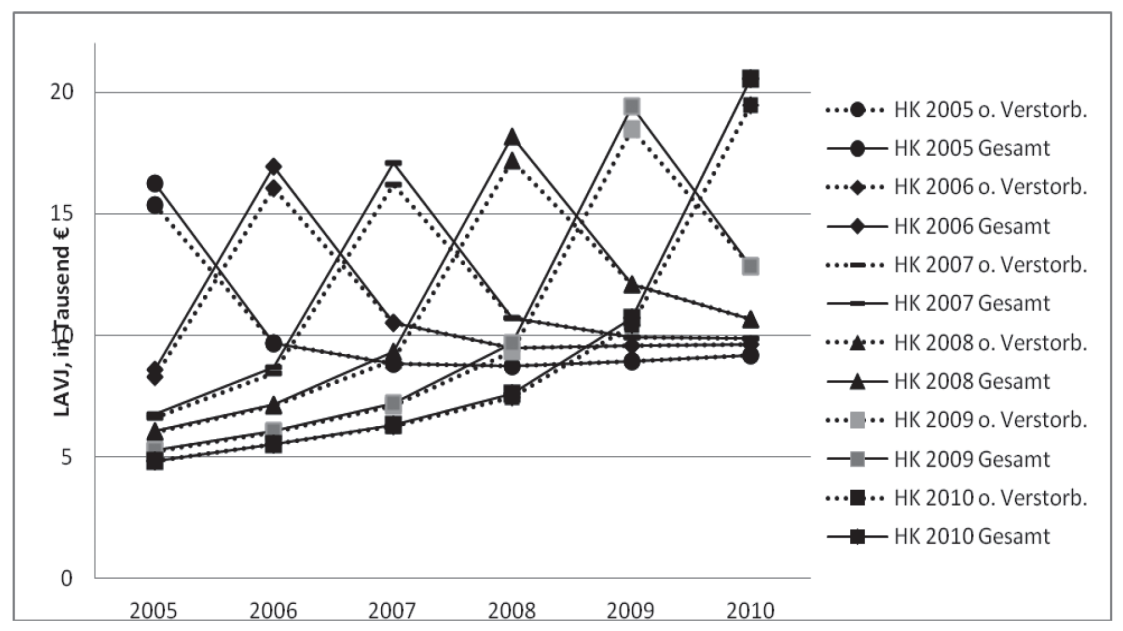

Quelle: Eigene Darstellung
Gesamtkohorten nur wenig (Abb. 4). Da jedoch auch Teilpopulationen mit deutlich höheren Sterberaten modelliert werden, wurden die Sterbefälle bei der weiteren Modellierung ausgeschlossen.

Dieser Umstand ist beachtenswert wegen der unten folgenden Diskussion in Abschnitt 7.4 über Veränderungsoptionen des RSA, zu denen auch die Annualisierung der Sterbekosten gehört. Der oben genannte Erhöhungsbetrag von $15.000 €$ pro Sterbefall ist allerdings nicht auf die RSA-Diskussion anwendbar, da es sich bei der Veränderungsoption des RSA nicht nur um Hochkostenversicherte handelt, sondern um durchschnittliche Sterbefälle, bei denen der Abweichbetrag bei der Annualisierung wesentlich niedriger ausfällt.

\section{Beitragsbedarf}

Für die Jahre 2009 und 2010 lagen für die Untersuchung Daten zum Beitragsbedarf (RSA-Einnahmen) vor. Für die Kohorten 2005 bis 2008 fehlen diese Informationen, da hier noch der alte RSA gültig war.

Vergleicht man in Tabelle 7 Beitragsbedarf und Leistungsausgaben der unterschiedlichen Kohorten in einem festen Jahr, hier 2009, so fällt auf, dass die Leistungsausgaben deutlich stärker variieren. Sie spreizen im Basisjahr 2009 von $9.003 €$ pro Kopf bei der Kohorte aus 2005 bis $19.493 €$ bei der Kohorte aus 2009, also über eine Spanne von über $10.000 €$. Die Beitragsbedarfe aus 2009 spreizen deutlich geringer: von $5.946 €$ bis $8.146 €$, also nur über $2.200 €$. Für Großgruppen zeigt sich der Verlauf des Beitragsbedarfes in Tabelle 7 gegenüber den Leistungsausgaben gleichmäßiger. Sogar nach einem Eskalationsjahr gibt es keinen auffälligen „Peak“, wie etwa bei den Leistungsausgaben. Während die Pro-Kopf-Ausgaben im Eskalationsjahr um ca. $10.000 €$ ansteigen, steigt der Beitragsbedarf im Folgejahr gerade einmal um ca. $1.000 €$ an. 
Mit den Anfang 2012 verfügbaren Daten ließen sich Deckungsbeiträge nur für die Jahre 2009 und 2010 darstellen. Verlässliche Daten für die Berechnung des Beitragsbedarfes stehen der Krankenkasse erst der zweiten Hälfte des Folgejahres zur Verfügung. Um dennoch längere Zeitreihen für Deckungsbeiträge zu erhalten und Aussagen zur Deckungssituation vor, während und nach einer Krankheitseskalation machen zu können, war also die Simulation von Beitragsbedarfen erforderlich. Da nur Betrachtungen von sehr großen Versichertengruppen erfolgen, war dabei keine Simulation individueller Beitragsbedarfe erforderlich und es konnte ein einfacher Ansatz gewählt werden.

\section{Zeitreihe der Deckungsbeiträge}

Mit diesen simulierten Beitragsbedarfswerten lässt sich unter Zuhilfenahme der vorliegenden Leistungsausgaben für jede Hochkostenkohorte für jedes Jahr ein Deckungsbeitrag berechnen, sodass man eine komplette 5-Jahres-Zeitreihe erhält.

Die Verlaufskurven der Deckungsbeiträge (Abb. 5) sind für jede Kohorte auffallend ähnlich. Die Kohorte, die 2010 in das Hochkostenstadium gekommen ist, weist gemäß dieser Simulation im Jahr 2005 noch positive Deckungsbeiträge auf, die sich in den nächsten Jahren verschlechtern, um 2010 den stark negativen Eskalationswert zu erreichen. Bei den anderen Kohorten ist dieser Verlauf je nach Lage des Eskalationsjahres mehr oder weniger nach links verscho-

\section{Abbildung 5: 5-Jahres-Zeitreihe der Deckungsbeiträge.}

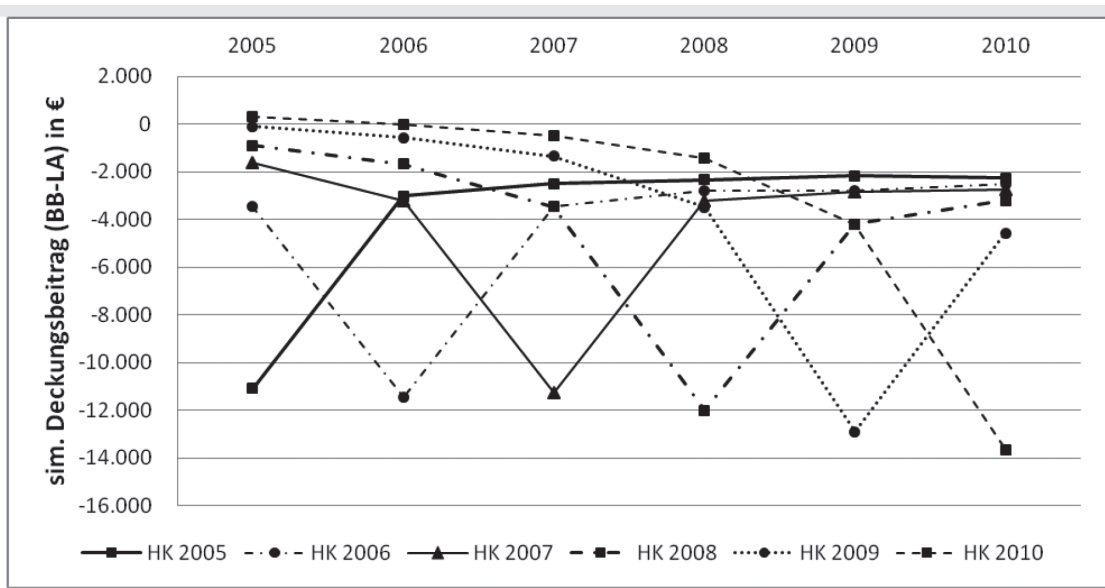

Quelle: Eigene Darstellung

ben. Die Hochkostenkohorte aus 2005 beginnt mit dem Eskalationsjahr und zeigt danach die Situation bis fünf Jahre nach der Eskalation. Dabei bleibt der Deckungsbeitrag unterhalb -2.000€ negativ (jährlich und pro Kopf). Hier ist zu bedenken, dass Sterbefälle ausgeschlossen wurden. Dadurch verbessert sich nach Drösler et al. (2011) die Deckung. Bei Einbeziehung der Sterbefälle wächst die Unterdeckung um ca. 400 Euro pro Kopf auf -2.400 Euro.

Durch die Korrektur der Inflation liegen die Kurven nahezu auf gleichem Niveau. Ab 2009 erkennt man allgemein „tiefer" liegende Werte. Dies hängt mit Veränderungen des Versichertenbestandes zusammen, die Einfluss auf das Ausgabenniveau der jeweiligen Hochkostenkohorte hatten.

Abbildung 6: Langzeitprofil der Deckungsbeiträge der Hochkostenversicherten.

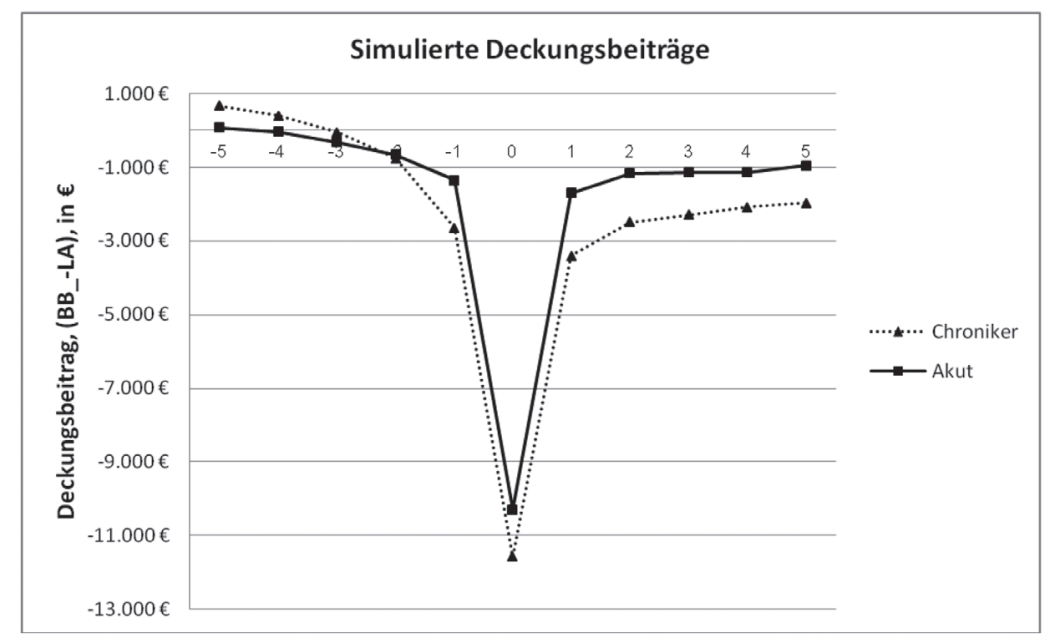

Quelle: Eigene Darstellung

\section{Das Langfristprofil der Deckungsbeiträge}

Diese sehr ähnlichen Verläufe ließen den Schluss zu, dass sie für Gruppen dieser Größe charakteristisch sind und sich zu einem Langfristprofil synthetisieren lassen. Dazu wurden die Mittelwerte der inflationsbereinigten Deckungsbeiträge gebildet, die jeweils den gleichen zeitlichen Abstand zum Eskalationsjahr der Leistungsausgaben hatten. Der Datenlage geschuldet liegen daher die meisten Informationen nahe des Eskalationsjahres vor und die wenigsten an den Rändern.

Abb. 6 zeigt das synthetisierte Langzeitprofil der Deckungsbeiträge. Der Verlauf beginnt mit beinahe ausgeglichenen Deckungsbeiträgen in der Zeit fünf Jahre vor Eskalation, fällt dann exponentiell bis zur Eskalation ab und steigt danach sprunghaft an, um auf einem Niveau unterhalb -2.000 Euro $\mathrm{zu}$ verbleiben. Aufgrund der wenigen Randwerte kann die Tendenz nicht klar interpretiert werden. Dass nach der Eskalation keine ausgeglichenen Werte erreicht werden, ist allerdings eindeutig, insbesondere unter der Berücksichtigung, dass Sterbefälle ausgeschlossen wurden (vgl. 6.2, bei Berücksichtigung der Sterbefälle sogar auf etwa -2.500 Euro). $\mathrm{Zu}$ beachten ist auch, dass der Deckungsbeitrag im Eskalationsjahr einen Wert von etwa -12.000 Euro annimmt. Bei der Größe der Gruppe von ca. 90.000 Versicherten entspricht das für die betrachtete Kasse einem permanenten Deckungsrisiko von über einer Milliarde Euro (bei einem Marktanteil in der GKV von ca. 3\%). 


\section{Untersuchung der Teilgruppen}

Die Bildung der Teilgruppen (Abschnitt 5.5 ) ist hier noch einmal zusammen gefasst:

1. Abspaltung: Versicherte unter einem Alter von 6 Jahren (Gruppe Infant)

2. Abspaltung: Versicherte mit Pflegestufe (Gruppe Pflege)

3. Abspaltung: Versicherte mit schwerwiegenden oder kostenintensiven chronischen Erkrankungen ${ }^{3}$ (Gruppe

4. Rest: In der Gruppe der restlichen Versicherten befinden sich überwiegend Akutfälle wie Infektionen und Unfälle und leichte chronische Erkrankungen (Gruppe Akut)

Die Langzeitreihen der Untergruppen zeigen unterschiedliche Verläufe (Abb. 8). Am auffälligsten ist die Gruppe „Infant“. Der Verlauf beginnt bei sehr niedrigen Werten für den Deckungsbeitrag. Die Werte liegen hier mehrjährig niedriger, als der Eskalationswert bei den übrigen Gruppen. Die Unterdeckung liegt bei der Gruppe „Infant“ im Eskalationsjahr unterhalb $-20.000 €$ pro Kopf. Danach steigt der Verlauf auf Werte um $-3.000 €$ an. Durch den hohen Anteil an Neugeborenen wird die Interpretation des Zeitraumes vor der Eskalation schwierig. Es handelt sich um eine kleine Restkohorte von Kleinkindern, die über Chroniker)

Abbildung 8: Langzeitreihen für die Untergruppen

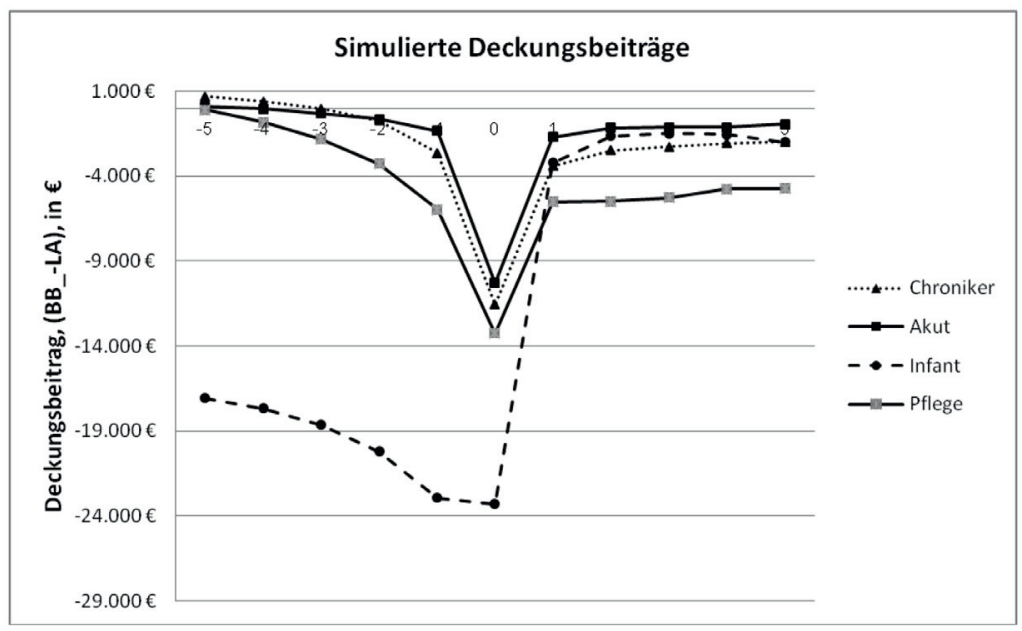

mehrere Jahre (meist seit der Geburt) einen sehr hohen Versorgungsbedarf hatten, dann aber durch ein (höchstwahrscheinlich medizinisches) Ereignis in eine Phase deutlich geringeren Versorgungsbedarfs traten. Ökonomisch betrachtet stellt diese Gruppe für eine Krankenkasse ein hohes und durch den RSA nicht gedecktes Ausgabenrisiko dar.

Auffällig ist auch der Verlauf der Gruppe „Pflege“. Abgesehen von der Gruppe „Infant“ weist die Gruppe „Pflege“ die schlechtesten Werte für den Deckungsbeitrag auf. Nach der Eskalation bleibt der Wert auf einem für eine Krankenkasse problematischen Niveau von $-5.000 €$.

Der Verlauf der Gruppe „Akut“ erweist sich erwartungsgemäß als der

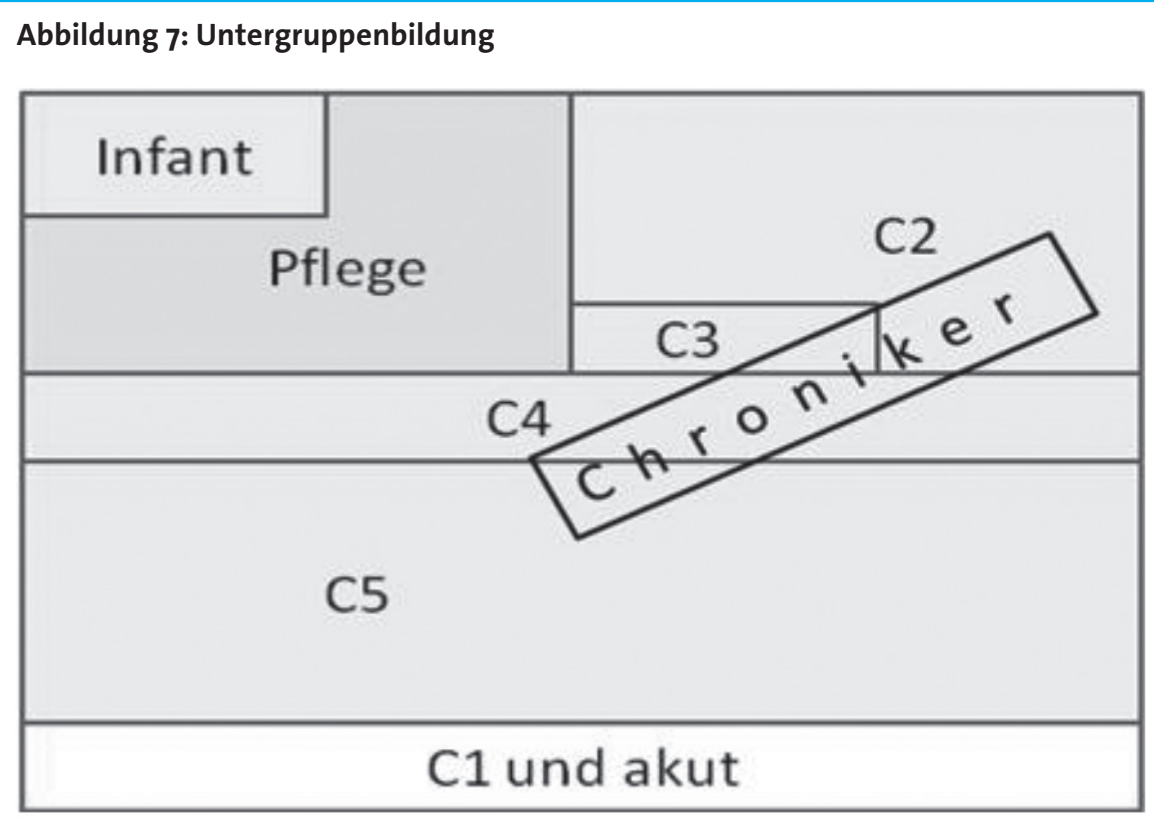

wirtschaftlich günstigste und erreicht nach der Eskalation als einziger annähernd ausgeglichene Werte.

Die Gruppe „Chroniker“ - aufgrund der Menge zu erwarten - ist weitgehend deckungsgleich mit dem Gesamtverlauf. $\mathrm{Zu}$ beachten ist, dass die Werte nach der Eskalation auf einem Niveau von etwa $-2.000 €$ verbleiben.

Schließlich wurden auch die Verläufe der detaillierten Chronikergruppen C2 bis C5 (vgl. Abschnitt 5.5) betrachtet (Abb. 9). Die kostenintensiven chronischen Erkrankungen C2 haben über den gesamten Zeitraum - abgesehen vom Eskalationsjahr - die ungünstigste Deckung. Ihre Ausgaben werden in diesem Vergleich am schlechtesten durch den Morbi-RSA gedeckt. Vor der Eskalation lässt sich eine deutliche Verschlechterung des Deckungsbeitrages beobachten. Das könnte für Prädiktionsmodelle interessant sein.

Ganz anders der Verlauf bei den schwerwiegenden chronischen Erkrankungen C3. Vor der Eskalation beobachtet man ausgeglichene, sogar positive Deckungsbeiträge, nach der Eskalation von allen Gruppen die geringste Unterdeckung. Die Eskalation erfolgt nahezu ohne Vorwarnung. Diese Gruppe ist allerdings sehr klein, daher schwankungsanfällig.

In der Gruppe C4 findet man die Kombination von C2- und C3-Erkrankungen vor (entweder oder). Man beobachtet vor der Eskalation - von anfangs

3 Es wurde die Definition des BVA-Krankheitsklassifikation gemäß der „schwarz-rotListe" verwendet. 
positiven Deckungsbeiträgen kommend - eine vergleichsweise moderate Verschlechterung. Nach der Eskalation verbleibt für die Krankenkasse ein markantes Deckungsproblem.

Schließlich zeigt sich noch ein sehr interessanter Verlauf für die Gruppe C5, also für die Personen, bei denen eine oder mehrere Erkrankungen vorliegen, die sowohl kostenintensiv als auch schwerwiegend sind. Die meisten dieser Erkrankungen sind in den HMG, also den berücksichtigten Krankheiten des RSA enthalten. Dies ist auch in der Phase vor der Eskalation an den anfangs deutlich positiven Deckungsbeiträgen zu erkennen. Die Eskalation erfolgt in dieser Gruppe finanziell gesehen am heftigsten. Überraschend ist, dass trotz der RSA-Relevanz dieser Erkrankungen der Ausgleich nach der Eskalation aus Kassensicht ähnlich problematisch ist, wie bei der Gruppe C2.

\section{Diskussion}

\section{Die Kostendeckung bei den Hochkostenversicherten}

Höhe und Verläufe der Leistungsausgaben bei Hochkostenversicherten waren im Vorfeld bekannt, überraschend sind die Erkenntnisse bei den Einnahmen aus dem Morbi-RSA, den Beitragsbedarfen. Bereits die vorliegenden Einnahmewerte aus 2009 und 2010 wiesen auf eine Unterdeckung bei diesen Versicherten hin. Die Beträge der Beitragsbedarfswerte sind in der Gesamtgruppe durchgehend deutlich niedriger, als die Beträge der Leistungsausgaben. Liegen die Deckungsbeiträge der Hochkostenversicherten fünf Jahre vor der Eskalation noch in der Nähe ausgeglichener Werte $(0 €$ Deckungsbeitrag), so liegen sie in den folgenden neun Jahren permanent darunter und addieren sich in diesen Jahren Verluste in Größenordnungen von $45.000 €$ pro Kopf. Welche Möglichkeiten haben Krankenkassen, diesem wirtschaftlichen Risiko durch Versorgungsansätze zu begegnen? Dafür ist es erforderlich, die in Abschnitt 6.6 vorgestellten Teilpopulationen einzeln zu diskutieren.

\section{Die Teilpopulationen}

Der Verlauf der Deckung bei der Chronikergruppe C5 (Abb. 9), also den Hochkostenversicherten, die häufig an einer

\section{Abbildung 9: Die Untergruppen der Chroniker $C_{2}$ bis $C_{5}$}

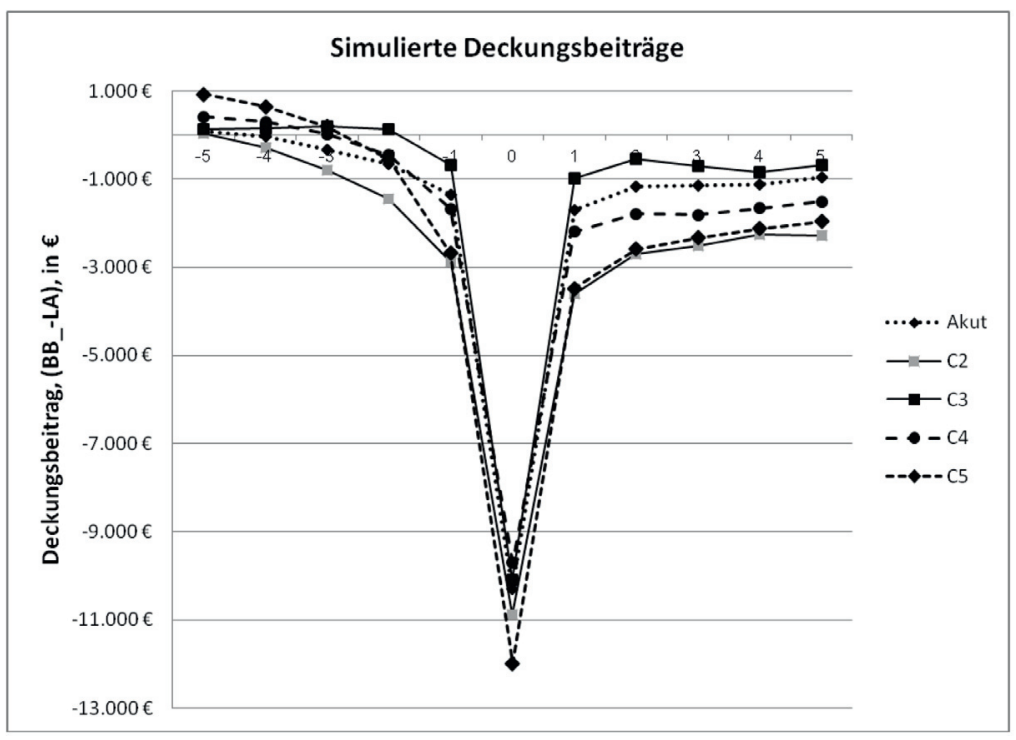

oder mehreren Erkrankungen leiden, die im Morbi-RSA besonders berücksichtigt werden, deutet am ehesten darauf hin, dass eine Vermeidung der Eskalation ökonomisch interessant sein könnte. Liegt hier der Schlüssel für den ökonomischen Erfolg der Versorgung durch die Krankenkasse? Welche Chancen hat sie konkret, diese Risikoversicherten zu identifizieren und in adäquate Versorgungsmodelle zu überführen? Sind diese Versicherten wirklich schlecht versorgt und warum? Weil Angebote fehlen? Oder weil der individuelle Lebensstil das Risiko mit sich bringt (Ernährung, Bewegung, Beruf)? Und falls ein Versorgungsangebot der Krankenkasse vom Versicherten angenommen wird, welchen Einfluss hat sie auf das Versorgungsgeschehen bei den Leistungserbringern? Die Erfahrungen, die in Abschnitt 7.3 zusammengefasst sind, dämpfen den Optimismus erheblich.

\section{Eine besonders problematische Gruppe sind offenbar Kleinkinder mit hohen Ausgaben.}

Eine besonders problematische Gruppe sind offenbar Kleinkinder mit hohen Ausgaben. Hier schlagen Frühgeburtenrisiken und angeborene Erkrankungen in Form von außerordentlich hohen Unterdeckungen durch. Ob diese hohen Ausgabenrisiken durch besondere Versorgungsprogramme einer Krankenkasse reduziert werden könnten, ist aufgrund der zu Grunde liegenden Erkrankungen bzw. Schädigungen mehr als fraglich. Diese Personengruppe kommt daher insbesondere für einen Ausgleich über einen Risikopool in Frage.

Diskussionswürdig ist auch die Gruppe der Hochkostenversicherten mit einer bestehenden Pflegestufe. Die Langzeitbetrachtung zeigt hier für die Zeit nach der Ausgabeneskalation vergleichsweise hohe Unterdeckungen. Die Versorgungsinitiativen der betrachteten Krankenkasse haben die Erkenntnis erbracht, dass eine Beeinflussung der Versorgung von Versicherten in Pflege wesentlich schwieriger ist, als bei Versicherten ohne Pflegestufe. Zu den logistischen Herausforderungen, die eine Versorgungsverbesserung ohnehin mit sich bringt, sind hier zusätzlich die Zusammenarbeit mit Angehörigen, Betreuern oder Pflegeeinrichtungen zu berücksichtigen. Erkenntnis ist auch, dass Erkrankungsverläufe bei Vergabe der Pflegestufe häufig bereits irreversibel sind. Versicherte mit Pflegestufen wären demnach im MorbiRSA ebenfalls gesondert auszugleichen.

\section{Ökonomisches Potenzial bei der Versorgungsverbesserung}

Über die jährlichen pro-Kopf-Einsparungen durch Ansätze „ambulant vor stationär" oder Telemedizin gibt es sehr unterschiedliche Zahlen. Einzelne Studien weisen eine deutliche Verringerung der stationären Aufenthalte und Nettoeinsparungen von über $5.000 €$ je 
Person und Jahr aus [Kissling 2008]. Andere Studien - vor allem bei den kostenintensiven psychiatrischen Krankheiten - zeigen selbst bei breit angelegten Langzeitstudien keine signifikanten Einspareffekte [Wittmann et al. 2011]. Monomorbidität und eindeutige (somatische) Krankheitsbilder wie z. B. Herzinsuffizienz scheinen wichtige $\mathrm{Vo}$ raussetzungen für den Erfolg von Versorgungsinitiativen $\mathrm{zu}$ sein [Kielblock et al. 2007]. Nolting (2012) weist aber darauf hin, dass Evaluationsergebnisse aufgrund der Wettbewerbssituation der Kassen nicht in ausreichendem Maße transparent sind. Gerlach et al. (2012) führen aus, dass bei der integrierten Versorgung selten eine Evaluation extern durchgeführt und dann publiziert wird.

In vielen Studien werden Verbesserungen der Lebensqualität [Nolting 2012] oder z. B. reduzierte Mortalitätsraten [Kielblock et al. 2007] nachgewiesen. Diese Art von Versorgungserfolgen ist jedoch nicht ökonomisch quantifizierbar und wird im Morbi-RSA nicht berücksichtigt.

Die in der untersuchten Krankenkasse erzielten Einsparungen im Gesundheitscoaching - nachgewiesen durch statistische Messverfahren mit Vergleichsgruppen - lagen in der Regel deutlich unter den o. a. Beträgen, trotz Vorauswahl der Patienten per Morbiditätsanalysen und einem individuell zugeschnittenen, intensiven Beratungsansatz. Die Versorgungsansätze umfassen seit 2007 die wesentlichen Risikoerkrankungen, bei denen Versorgungsexperten eine Beeinflussung für möglich halten. Darunter fallen z. B. Herzinsuffizienz, Diabetes, koronare Herz-Kreislauferkrankungen, COPD, Depression und Schizophrenie. Es konnte bislang lediglich ein (erwarteter) Anstieg bei Arzneimittelausgaben in der psychiatrischen Versorgung signifikant nachgewiesen werden. Der Erfolg von Versorgungsinitiativen hängt nach Erfahrungen in der untersuchten Krankenkasse stark von der Teilnahme- und Mitwirkungsbereitschaft der Versicherten ab. Vorab waren Investitionen in siebenstelliger Höhe notwendig. Diese werden vom Morbi-RSA nicht ausgeglichen.

Die zur wirtschaftlichen Evaluation angewandten Messmethoden sind nach den Erfahrungen der Autoren im Allgemeinen sehr manipulationsanfällig. In einzelnen Studien wird nicht die Ausgabeneinsparung ausgewiesen, sondern die Verbesserung der Deckungsbeiträge [Rabatta 2009]. Diese stellt sich ein, sobald vom Arzt mehr Diagnosen codiert werden und dadurch mehr Zuweisungen aus dem Morbi-RSA fließen. Inwiefern das einen Versorgungserfolg darstellt, kann bezweifelt werden.

In den letzten Jahren ist verbreitet ein Rückgang der integrierten Versorgung zu beobachten. Auf Fachkonferenzen der GKV werden regelmäßig die fehlenden wirtschaftlichen Anreize diskutiert. Seit 2008 stagnieren die Zahlen [Gerlach et al. 2012].

Einen weiteren Versorgungsweg stellen die Disease-Management-Programme (DMP) dar. Dabei handelt es sich um strukturierte Behandlungsprogramme für die am weitesten verbreiteten chronischen Erkrankungen wie Brustkrebs, Diabetes, Asthma/COPD und koronare Herzerkrankungen mit einer dezidierten, gesetzlich geregelten Dokumentationspflicht für die behandelnden Ärzte. 2012 berichtet der Sachverständigenrat zur Begutachtung der Entwicklung im Gesundheitswesen [Gerlach et al. 2012], dass bei mittlerweile 5,9 Mio. eingeschriebenen Versicherten in fast 11.000 Programmen trotz ,verpflichtender Evaluation keine repräsentativen und validen Belege für eine Effizienz der strukturierten Behandlungs- bzw. Disease-Management-Programme“ vorlägen“. Für die DMP-Programme erhalten die Krankenkassen Geldzuweisungen aus dem Gesundheitsfonds. 2011 betrugen diese Zuweisungen für DMPProgramme für die GKV insgesamt ca. 1 Milliarde EUR [BMG 2012].

\section{Schlussfolgerungen}

Selbstverständlich können dies nur Indizien für eine Verteilungsproblematik im Morbi-RSA sein, denn es gibt auch Versichertengruppen, die über längere Zeiträume hohe Überdeckungen haben. Dazu zählen ältere Versicherte mit Diagnosen, die krankheitsbedingte Zuweisungen verursachen, bei denen aber keine höheren Leistungsausgaben anfallen. Solange die Gruppen mit systematischer Überdeckung mit solchen mit systematischer Unterdeckung sich im Versichertenbestand in Waage halten, stellt dies für die Krankenkasse kein direktes finanzielles Problem. Schwierig wird es, wenn ein Versichertenbestand ein einseitiges Ungleichgewicht aufweist.

Weiter führende Analysen haben ergeben, dass insbesondere der Leistungsbereich Krankengeld unternehmensspezifische Deckungsprobleme mit sich bringt. Im Unterschied zum krankheitsbezogenen Ausgleich z. B. bei wie Krankenhaus- oder Arztleistungen, wird beim Krankengeld der Beitragsbedarf wie im alten RSA vor 2009 nur nach Alter und Geschlecht ausgeglichen (vgl. Abschnitt 3). Die stärksten Ausgabenentwicklungen sind bei den Krankengeldzahlungen für psychische Erkrankungen zu verzeichnen. Aus der Versorgungssicht bringt dieser Tatbestand besondere Probleme mit sich, denn eine Krankenkasse ist bei allen Versorgungsansätzen auf die Mitwirkungsbereitschaft der Versicherten angewiesen. Gerade Versicherte mit

\section{Ein direkter Ausgleich der Krankengeldausgaben wäre eine Option zur Korrektur des Morbi-RSA: die unterschiedliche Deckungssituation der Krankenkassen beim Krankengeld würde beseitigt.}

schwerwiegenden psychischen Erkrankungen lassen sich jedoch häufig nicht in Versorgungsprogramme einbinden. Die Quoten nicht erreichbarer oder sich verweigernder Personen sind hier höher, als bei somatischen Erkrankungen. Nach Informationen des BVA [Göpffarth 2011] streut die Deckung beim Krankengeld von unter $60 \%$ bis über $155 \%$ bei den Krankenkassen. Diese Streuung wird durch kein anderes Berechnungsverfahren beseitigt. Ein direkter Ausgleich der Krankengeldausgaben wäre daher eine Option zur Korrektur. Er würde zumindest die sehr unterschiedlichen Deckungssituationen der Krankenkassen beim Krankengeld beseitigen. Auf der anderen Seite ginge damit ein Anreiz zur Fallprüfung und -steuerung verloren. Diesem Aspekt ließe sich z. B. durch einen 95\%-Ausgleich begegnen.

Unter den Experten des Morbi-RSA wird derzeit die Annualisierung der Sterbekosten diskutiert. Zur Zeit gehen nur die tatsächlich angefallenen Ausgaben von Verstorbenen in die Berechnung der RSA-Zuweisungen ein. Eine Annuali- 
sierung würde zu einer Erhöhung der altersbezogenen Anteile für Hochbetagte führen und zu einer Erhöhung von Krankheitszuschlägen, die vor allem alte Menschen betreffen. Bei anderen Gruppen - vor allem die die Jüngere betreffen - käme es zu Kürzungen der Zuweisungen. Methodisch - so die RSA-Gutachter - wäre die Annualisierung eine Verbesserung [Drösler et al. 2011]. Allerdings gibt es aufgrund der Konstruktion des Morbi-RSA bereits heute eine gewisse systematische Bevorteilung älterer Versichertenbestände. Diese ergibt sich aus der Definition der Zuweisungen für Morbiditätsgruppen, z. B. über die Zusammenfassung der Diagnosen und die Fallzahlgewichtung [Batta 2012]. Sicher ist, dass die Annualisierung der Sterbekosten eine Verteilungswirkung von Einnahmen von jüngeren zu älteren Versicherten hätte.

Daher sei noch einmal darauf hingewiesen, dass in Abschnitt 6 Untersuchungsergebnisse von Hochkostenkohorten ohne Sterbefälle dargestellt sind, die festgestellten Unterdeckungen folglich nicht durch Sterbefälle verursacht werden und dass daher durch Annualisierung der Sterbekosten das aufgezeigte Problem nicht beseitigt wird.

Als sinnvolle Ergänzung zum MorbiRSA wäre ein Risikopool denkbar. Er ließe sich einschränken auf Erkrankungen, bei denen für die Kassen keine systematischen Beeinflussungsmöglichkeiten der Versorgung bestehen. Interessant in diesem Kontext sind sicher die in $\mathrm{Ab}$ schnitt 7.2 aufgeführten Teilgruppen. Der Schwellenwert der Leistungsausgaben sollte nicht zu niedrig liegen, damit eine effiziente Fokussierung auf die auszugleichenden Risiken stattfindet. Der Rückerstattungsbetrag sollte allerdings dann nicht zu knapp bemessen sein und mindestens $70 \%$ der Ausgaben betragen.

Schließlich wäre auch eine Ausweitung auf alle Erkrankungen denkbar. Drösler et al. (2011) führen allerdings aus, dass die Effekte vergleichsweise gering wären. Dagegen spricht der Aufwand, der für die 286 hinzutretenden Krankheitsgruppen (366, mit denen die rd. 15.000 Diagnosen des ICD-10 vollständig abgebildet werden, abzüglich der 80, die 2009 für den Morbi-RSA ausgewählt wurden) betrieben werden müsste.

Der Sachverständigenrat wirft $\mathrm{zu}$ Recht die Frage auf, warum die DMPProgramme nach wie vor eine eigen- ständige gesetzliche Versorgungsform sind. Jährlich bekommen die Krankenkassen aus dem Gesundheitsfonds ca. eine Milliarde Euro, die sie an die Ärzte weitergeben, ohne dass bislang ein Effizienznachweis vorliegt [Gerlach et al. 2012]. Verursacht dieser Umstand auch einen Teil der oben aufgezeigten Unterdeckung? Wäre es nicht auch eine Option zur Änderung des RSA, dieses Geld in mehr Erfolg versprechende Versorgungsansätze zu leiten?

\section{Hohe Leistungsausgaben können von Krankenkassen durch Versorgungsansätze nicht oder nur unwesentlich reduziert werden.}

Insgesamt betrachtet zeigen die Untersuchungsergebnisse die Grenzen der Möglichkeiten einer Krankenkasse auf, die wirtschaftliche Situation durch Versorgung von Hochkostenversicherten zu verbessern. Die hohen Leistungsausgaben können durch Versorgungsansätze nicht oder nur unwesentlich reduziert werden. Die Einsparungen je Versichertem sind deutlich geringer, als die aufgezeigten permanenten Unterdeckungen.

Erschwerend dazu kommen die hohen Hürden, die der Gesetzgeber - als verlängerter Arm das Bundesversicherungsamt - den Krankenkassen für Versorgungsmodelle auferlegt hat. Kosteneinsparungen müssen praktisch von vorneherein nachgewiesen werden [Gerlach F. et al., 2012]. Die „Anschubfinanzierung“ gibt es seit 2009 nicht mehr.

Betrachtet man den Gesundheitsfonds volkswirtschaftlich, so stellt man eine Fehlentwicklung fest: Die Beiträge werden von allen GKV-Mitgliedern eingezahlt. Überschüsse erzielen allerdings vor allem Kassen mit überdurchschnittlich gesunden Versicherten. Dieser Umstand wird von den RSA-Gutachtern als wesentliches Ergebnis herausgestellt [Drösler et al. 2011]. ${ }^{4}$ Überschusskassen werden nun vom Gesetzgeber genötigt, eine Prämie an ihrer Mitglieder auszuzahlen. Somit fließt das Geld makroskopisch betrachtet permanent von den Kranken zu den Gesunden. Investitionen in Versorgungsverträge und höhere Personalausgaben für Beratung und Betreuung Kranker werden mit Unterdeckung bestraft. Damit wird das Ziel des Morbi-RSA - die effiziente und qualitative Versorgung [Bohlen-Schöning, 2008] - noch nicht erreicht worden.

\section{Fehlerabschätzung}

Unsicher in der Modellierung der Deckungsbeiträge ist vor allem der Beitragsbedarf. Die Leistungsausgaben liegen als reale Ist-Kosten für hinreichend große Mengen vor. Die Perioden sind so abgegrenzt, dass die Ausgaben vollständig vorlagen. Bestimmte Ausgaben wurden pauschalisiert, z. B. die Ausgaben für ambulante Arztbehandlung. Im Einzelnen können dadurch Unterschiede zu den morbiditätsbezogenen Individualkosten von ca. $500 €$ auftreten. Die Ausgaben der Hochkostenversicherten werden durch die Pauschalisierung eher entlastet.

Der Beitragsbedarf wurde durch das Grouping individualisiert und mit den Gesamtwerten des Versichertenbestandes adjustiert. Für die betrachteten Gruppen und Untergruppen kann der dadurch auftretende Fehler nicht abgeschätzt werden. Bei Kleingruppen (z. B. C3) ist es aber sicher nicht zu vernachlässigen, besonders dann nicht, wenn er systematisch auftritt.

Die Preissteigerungen bei den Leistungsausgaben betragen in den Jahren 2005 bis 2010 zwischen 4 und $6 \%$. Die Inflation wurde mit $5 \%$ bereinigt. Der Fehler beträgt daher im Mittel 0,5\%, das sind ca. $50 €$.

Die Annualisierung der Leistungsausgaben bei Versicherten, die weniger als 365 Tage im Bestand waren weist einen Hochrechnungsfehler auf. Dieser wird mit $300 €$ abgeschätzt.

Die Morbiditätsveränderung des Versichertenbestandes überlagert die einzelnen Phasen des Deckungsbeitragsverlaufes. Mit dem Vergleich der Kohorten lässt sich abschätzen, dass dieser Effekt im Jahr $2009329 €$ und im Jahr $2010150 €$ Erhöhung der Ausgaben ausmacht.

4 „Für „Gesunde“ und Versicherte, die geringe Ausgaben verursachen, bestehen umgekehrt nach wie vor spürbare Überdeckungen durch die Zuweisungen aus dem Gesundheitsfonds." 


\section{Literatur}

Batta, Dr. E. 2012; Effekte der Krankheitsauswahl 2013 in Konferenz „Dauerbaustelle Morbi-RSA“ 26.4.2012, Essen.

BMG 2012, Bundesministerium für Gesundheit, amtliche Statistik der GKV KJ 1 für 2011 Berlin.

Bohlen-Schöning, S., 2008: Morbi-RSA sinnvolle Weiterentwicklung des bisherigen RSA, in Zentrum für Sozialpolitik Bremen, Gesundheitspolitisches Kolloquium 3.12.2008, BVA, Bonn.

BVA 2008: So funktioniert der neue Risikostrukturausgleich im Gesundheitsfonds, Bonn 2008

BVA 2010, Festlegung der im RSA zu berücksichtigenden Krankheiten durch das BVA (30.06.2010) nach § 31 Abs. 4 Satz 1 RSAV, Internetseite des Bundesversicherungsamtes

BVA 2012, Festlegungen nach $\S 31$ Abs. 4 RSAV für das Ausgleichsjahr 2013

(28.09.2012), Internetseite des Bundesversicherungsamtes

Drösler, S.; Wasem, J. et al. (2011): Evaluationsbericht zum Jahresausgleich $2009 \mathrm{im}$ Risikostrukturausgleich.

Gerlach, F. et al. (2012): Wettbewerb an der Schnittstelle zwischen ambulanter und stationärer Gesundheitsversorgung, Sondergutachten SVR, Bundesdrucksache 17/10323, Bonn

Göpffarth, D. (2011); Aktuelle Entwicklungen beim Risikostrukturausgleich, AG Finanzen und Risikomanagement bei den Gesundheitsforen Leipzig am 10.5.2011.

Kielblock, B. et al. (2007); Einfluss einer telemedizinisch unterstützten Betreuung auf Gesamtbehandlungskosten und Mortalität bei chronischer Herzinsuffizienz, DMW 2007, 132, S. 417-422.

Kissling, W. (2008): Das Münchner Modell - Krankenkassen finanzieren ComplianceProgramme, Psychoneuro, 34(9): 410-415.

Nolting, H.-D., Hackmann, Dr. T. (2012); Bestandsaufnahme von komplexen lokalen, regionalen und überregionalen sektorübergreifenden Modellprojekten zur Versorgung von Menschen mit psychischen Erkrankungen, Berlin 2012

Rabatta, S. (2009): Integrierte Versorgung: Kooperation im Kinzigtal spart Kosten. In: Deutsches Ärzteblatt 106 (20), S. Ag66-A968.

RSAV 1994. Risikostrukturausgleichsverordnung. BGBI. I S.55, Stand 2012 BGBI. 1601

Schmidt, D.; Göpffahrt, D. (2009:): Die Berücksichtigung von Verstorbenen im Morbi-RSA. In: Barmer/GEK: Gesundheitswesen aktuell 2009

Schmidt, U. 2009: Interview im „WeserKurier" am 14.08.2009

Wille, Dr. E., Thüsing, Dr. G. (2012): Wettbewerb an der Schnittstelle zwischen ambulanter und stationärer Gesundheitsversorgung. Sondergutachten SVR Gesundheit, Bonn.

Wittmann, W. W. et al. (2011): Qualitätsmonitoring in der ambulanten PsychotherapieAbschlussbericht 2011, Hamburg 2011.

\section{Gesundheitstourismus}

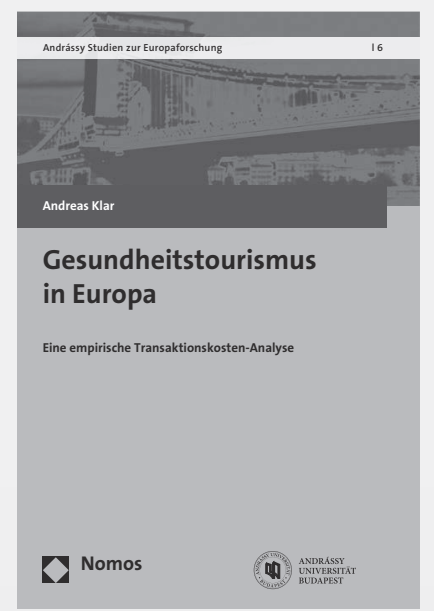

\section{Gesundheitstourismus in Europa}

Eine empirische Transaktionskosten-Analyse

Von Andreas Klar

2013, 287 S., brosch., 49,- €, ISBN 978-3-8487-0314-2

(Andrássy Studien zur Europaforschung, Bd. 6)

Die Inanspruchnahme von medizinischen Leistungen im Ausland („Gesundheitstourismus“) wird seit Jahren als Trend und Wachstumsfeld dargestellt. Das Inkrafttreten der EU-Richtlinie zur grenzüberschreitenden Gesundheitsversorgung im April 2011 ist nur ein Beispiel für diese zunehmende Bedeutung von Auslandsbehandlungen.

Gleichzeitig herrscht ein Mangel an empirischen Analysen über Patienten, die sich im Ausland medizinisch haben behandeln lassen. Warum sind sie ins Ausland gefahren? Welche Behandlungen haben sie im Ausland durchführen lassen? Welche Kosten sind ihnen dabei entstanden - monetär und nicht-monetär in Form von Transaktionskosten? Wie kann die Qualität von Auslandsbehandlungen bewertet werden?

Diese Fragen werden anhand empirischer Daten von über 6.000 deutschen Patienten, die sich im Ausland haben behandeln lassen, sowie anhand von über 300 internationalen Zahnpatienten in Budapest beantwortet.

Weitere Informationen unter: www.nomos-shop.de/20627

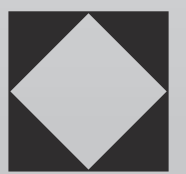

